\title{
Performance Analysis Based on RAU Selection and Cooperation in Distributed Antenna Systems
}

\author{
Gang Wang ${ }^{1}$, Chao Meng ${ }^{2}$, and Wei Heng ${ }^{1, *}$ \\ ${ }^{1}$ National Mobile Communication Research Laboratory, Southeast University \\ Nanjing, 210096, China \\ [e-mail: wanggang@seu.edu.cn,wheng@seu.edu.cn] \\ ${ }^{2}$ School of Communication Engineering, Jinling Institute of Technology \\ Nanjing, 211169, China \\ [e-mail: mengchao@jit.edu.cn] \\ *Corresponding author: Wei Heng
}

Received October 26, 2017; revised May 8, 2018; accepted September 6, 2018; published December 31, 2018

\begin{abstract}
In this paper, the downlink performance of multi-cell distributed antenna systems (DAS) with a single user in each cell is investigated. Assuming the channel state information is available at the transmitter, four transmission modes are formulated as combinations of remote antenna units (RAUs) selection and cooperative transmission, namely, non-cooperative transmission without RAU selection (NCT), cooperative transmission without RAU selection (CT), non-cooperative transmission with RAU selection (NCT_RAUS), and cooperative transmission with RAU selection (CT_RAUS). By using probability theory, the cumulative distribution function (CDF) of a user's signal to interference plus noise ratio (SINR) and the system ergodic capacity under the above four modes are determined, and their closed-form expressions are obtained. Furthermore, the system energy efficiency (EE) is studied by introducing a realistic power consumption model of DAS. An expression for determining EE is formulated, and the closed-form tradeoff relationship between spectral efficiency (SE) and EE is derived as well. Simulation results demonstrate their consistency with the theoretical analysis and reveal the factors constraining system EE, which provide a scientific basis for future design and optimization of DAS.
\end{abstract}

Keywords: distributed antenna systems, remote antenna unit, cooperative transmission, ergodic capacity, power consumption, energy efficiency, spectral efficiency

This work is supported in part by the National Natural Science Foundation of China (61771132, 61471115), by the Natural Science Foundation of the Higher Education Institutions of Jiangsu Province (16KJB510011), by the 2016 Science and Technology joint research and innovation foundation of Jiangsu province (BY2016076-13), by the Research Fund of National Mobile Communications Research Laboratory, Southeast University (No.2018A02), by the Research Foundation of Jinling Institute of Technology for Advanced Talents (40620044). 


\section{Introduction}

$\mathbf{T}_{\mathrm{o}}$ meet the requirements of high-speed data and multimedia services, wireless communication systems are evolving toward maximum data rates. Multiple input multiple output (MIMO) is considered one of the key technologies in next-generation mobile communication systems. It can improve spectrum efficiency (SE) without additional power and bandwidth consumption [1]-[3]. Antennas are deployed at the center of a cell in traditional cellular networks. Compared to traditional cellular networks, the distributed antenna system (DAS) can reduce transmission power, expand cell coverage, improve system SE, and this system has been studied widely [4], [5]. The concept of DAS has been written into the standard of LTE-A [6], and a few practical problems related to the design of DAS have been studied as well [7], [8].

In DAS, several remote antenna units (RAUs), also called remote radio heads (RRHs) in Long Term Evolution (LTE), are distributed in a cell and connected with the baseband processing unit (BPU) through an optical fiber connection. The RAUs are used only to transmit and receive signals, and the signals are processed in the BPU by using advanced signal processing techniques. Because there is no need for complex signal processing at the RAUs, it is helpful to reduce the size and cost of RAUs. A macroscopic multiple input single output (MISO) vector channel can be constructed using multiple RAUs [9], [10]. Efforts have been made to improve the performance of DAS by using geographically separated RAUs, such as the design of RAU locations [11]-[13]. Early studies mainly focused on the uplink performance [14], [15]. The downlink performance of DAS was analyzed from the viewpoint of information theory in [16], [17].

With the introduction of space diversity, cooperative transmission can increase the system performance significantly. Therefore, cooperative transmission has attracted considerable attention in wireless communications. In DAS, when the user is far from the RAU, cooperative transmission can effectively improve the user's signal to interference plus noise ratio (SINR), thus reducing the system outage probability. Multiple RAUs are connected via optical fibers and do not require additional radio relay resources for cooperative transmission. To maximize the ergodic sum rate, the cooperative transmission strategy by using pairings of distributed antenna ports and users in a single-cell downlink system was studied [18]. Although the user's SINR and system capacity were considered, the system energy efficiency (EE) was not considered in [18]. Cooperative transmission and selection diversity were considered to analyze the downlink capacity of multi-cell DAS [19]. In the cooperative transmission mode, all RAUs constituted a macro multi-antenna system, and the signal was transmitted through all RAUs. In the RAU selection diversity mode, considering the minimization of propagation path loss as a standard, only one RAU was selected for signal transmission. However, cooperative transmission and selection diversity were not jointly considered in [19]. The tradeoff between SE and EE in downlink DAS was studied in [20]-[22], in which all RAUs transmitted the signals to multiple users, but RAU selection transmission was not considered. In [23], all RAUs cooperated with each other to serve users, and the users' channels were orthogonal to each other. A power allocation scheme for system EE maximization was proposed, and per-antenna power constraint and proportional data rates for users were considered. The ergodic capacity and outage performance of DAS with imperfect channel state information (CSI) were analyzed in [24], and the corresponding accurate closed-form 
expressions were derived. However, it only considered RAU selection. RAU cooperative transmission and system EE were not considered. A downlink single-cell DAS with a single user was considered in [25]. A heuristic RAU selection method based on the distance from RAUs to the user to improve the EE of DAS was proposed, but the theoretical derivation was not given. A single-cell downlink DAS with multiple RAUs and one user was considered in [26]. A power allocation problem involving the maximization of EE under the minimum rate constraint was proposed. The optimal number of active RAUs was determined, and an optimal power allocation scheme in closed-form was obtained. Downlink power control in massive MIMO networks with distributed antenna arrays was investigated in [27]. A max-min power control algorithm to ensure each user gets uniform quality of service was proposed. The EE of DAS-based simultaneous wireless information and power transfer was studied in [28], where a power-splitting scheme was applied to Internet of Things devices. In addition, an optimal closed-form solution based on the Karush-Kuhn-Tucker (KKT) conditions was presented. However, few studies have investigated the SE and EE of DAS concurrently considering the combination of RAUs selection and cooperative transmission, which is the motivation underlying the present work.

In this work, the cumulative distribution function (CDF) of users' SINR, ergodic capacity, and system EE are studied in a multi-cell downlink DAS scenario. According to the combinations of RAU selection and cooperative transmission, four transmission modes, namely, non-cooperative transmission without RAU selection (NCT), cooperative transmission without RAU selection (CT), non-cooperative transmission with RAU selection (NCT_RAUS), and cooperative transmission with RAU selection (CT_RAUS), are formulated. When the selected active RAUs transmit signals to the user, the other RAUs in the same cell are assumed to be in the closed state. The main contributions of the present study are as follows:

(1) By using the CSI from the RAUs to the user and the probability theory, the CDF of the users' SINR and the ergodic capacities of various transmission modes are first derived theoretically. Then, closed-form expressions are developed. The Monte Carlo simulation results agree well with the theoretical derivations.

(2) By introducing a detailed power consumption model of DAS, system EE is studied. Then, the tradeoff relationship between SE and EE is deduced. The specific simulation results provide a scientific basis for future design and optimization of DAS.

The remainder of this paper is organized as follows. The system model is described in Section II. A transmission strategy based on RAU selection and cooperation is proposed in Section III. In Section IV, performance analysis of user's SINR and ergodic rate are performed. In Section V, a power consumption model of DAS is introduced, and then, the tradeoff relationship between SE and EE is derived. The simulation results are given in Section VI. Finally, a few concluding remarks are given in Section VII.

\section{System Model}

Consider a multi-cell downlink DAS with a seven-cell structure, as shown in Fig. 1, where each cell is surrounded by one continuous tier of six cells. A single user scenario in each cell is assumed, and this assumption is reasonable for most practical multi-user systems, such as orthogonal frequency division multiple access (OFDMA) [29]. The number of RAUs in each cell is $M=7$. The user and each RAU are configured with a single antenna. It is assumed that when active RAUs in each cell transmit signals to the user over the same frequency band, the other RAUs in the cell are closed [30]. Hence, the target user in each cell will face interference 
from active RAUs in the other six surrounding cells. Without loss of generality, we denote the cell in the center of each seven-cell structure as cell-0 and the other surrounding cells as cell-c. Thus, the signal received by the target user in cell-0 can be expressed as follows:

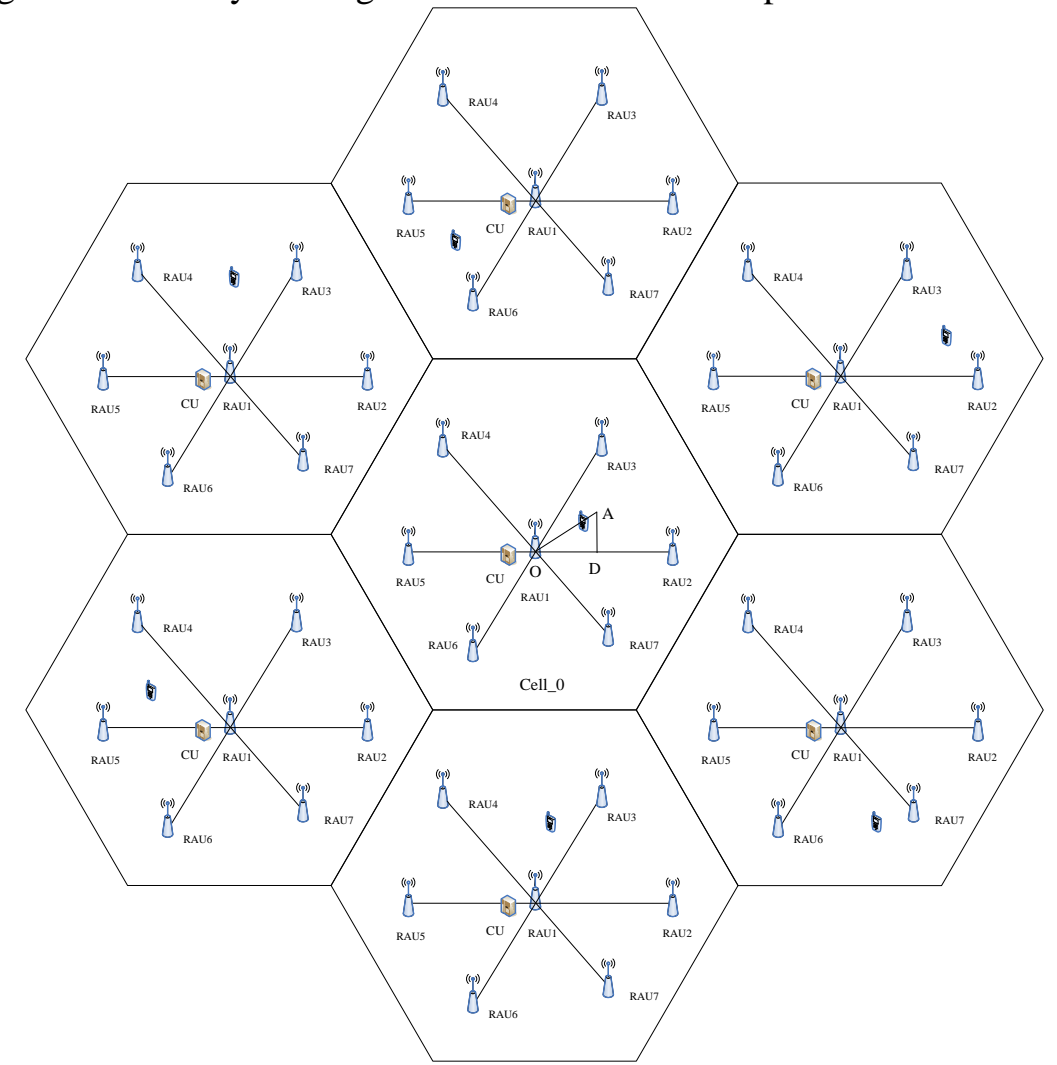

Fig. 1. Network model

$$
y=\sum_{l=1}^{L} \sqrt{P_{l} S_{l}} h_{l} x_{l}+\sum_{c=1}^{6} \sum_{j=1}^{L_{c}} \sqrt{P_{c j} S_{c j}} h_{c j} x_{c j}+z
$$

where $P_{l}$ (resp. $P_{c j}$ ) is the transmission power of the $l$-th RAU in cell-0 (resp. the $j$-th RAU in cell-c). It is assumed that all RAUs have the same transmission power, that is, $P_{l}=P_{c j}=P . L$ (resp. $L_{c}$ ) is the number of the active cooperative RAUs in cell-0 (resp. cell-c). $S_{l}=d_{l}^{-\alpha}$ (resp. $S_{c j}=d_{c j}^{-\alpha}$ ) denotes the path loss, where $\alpha$ is the path loss exponent value, and $d_{l}$ (resp. $d_{c j}$ ) is the distance from the $l$-th RAU in cell-0 (resp. the $j$-th RAU in cell-c) to the target user. $h_{l}$ (resp. $h_{c j}$ ) denotes the gain of the Rayleigh fading channel, which is modeled using an independent identically distributed (i.i.d) complex Gaussian random variable with zero mean and unit variance. $x_{l}$ (resp. $x_{c j}$ ) is the transmitting symbol of the $l$-th RAU in cell-0 (resp. the $j$-th RAU in cell-c), and the average power is $\mathbb{E}\left[\left|x_{l}\right|^{2}\right]=1$ (resp. $\mathbb{E}\left[\left|x_{c j}\right|^{2}\right]=1$ ). $z$ is the additive white Gaussian noise (AWGN) with mean zero and variance $\sigma_{z}^{2}$. Therefore, the interference plus noise power can be given as $\sigma_{I z}^{2}=\sum_{c=1}^{6} \sum_{j=1}^{L_{c}} P S_{c j}+\sigma_{z}^{2}$. Let the average received signal power to interference plus noise power ratio from the $l$-th RAU to the target user be $\lambda_{l}=P S_{l} / \sigma_{I I}^{2}$. 


\section{Transmission Strategy Based on RAU Selection and Cooperation}

\subsection{Transmission Strategy without RAU Selection}

In the transmission strategy without RAU selection, it is assumed that the RAUs transmitting the desired signals are determined by $\lambda_{1}$, and they do not change based on the instantaneous CSI. Therefore, the RAU with the largest $\lambda_{l}$ is selected in the NCT mode, and the two RAUs with the largest and the second-largest $\lambda_{l}$ are selected in the CT mode. For simplicity of analysis, we assume $\lambda_{1}$ and $\lambda_{2}$ are the two largest value, and $\lambda_{1}>\lambda_{2}$.

In the traditional NCT mode, a single fixed RAU transmits signals to the served user, regardless of the channel conditions. When RAU1 transmits a signal to the user, the corresponding SINR expression can be written as follows:

$$
\gamma_{\mathrm{NCT}}=\frac{P S_{1}\left|h_{1}\right|^{2}}{\sigma_{I z}^{2}}
$$

When the user is close to RAU1, the user's SINR increases.

According to the CSI available at the transmitter, in the CT mode, RAUs can cooperate in the opened-loop mode or the closed-loop mode. In the opened-loop mode, space time block codes (STBC) can be used for cooperative transmission. In the closed-loop mode, equal gain transmission (EGT) and maximum ratio transmission (MRT) are suitable for cooperative transmission. In the following statement, it is assumed that the transmitter has known CSI, and the MRT mode is used for cooperative transmission [31]. When RAU1 and RAU2 cooperatively transmit signals to the target user, the user's SINR can be expressed as follows:

$$
\gamma_{\mathrm{CT}}=\frac{P S_{1}\left|h_{1}\right|^{2}+P S_{2}\left|h_{2}\right|^{2}}{\sigma_{I z}^{2}}
$$

From (2) and (3), in a transmission strategy without RAU selection, the user's SINR with cooperative transmission is always better than that without cooperative transmission, that is, $\gamma_{\mathrm{CT}}>\gamma_{\mathrm{NCT}}$.

\subsection{Transmission Strategy with RAU Selection}

As for the transmission with RAU selection, the RAUs transmitting the desired signal to the target user can be selected according to the instantaneous CSI. Based on the real-time CSI from RAUs to the user, a few RAUs are selected to transmit signals to the user, and the user's rate can be further improved. When the user is at the coverage edge of the RAUs, in a certain time slot, the signals from the RAUs tend to be transmitted under better channel conditions. $N$ transmission sources are selected from $L$ candidate transmission sources for cooperative transmission, and the number of corresponding possible combinations is $K_{N}=C_{N}^{L}$. The RAUs set in the $k$-th combination is represented as $D_{k}, k=1, \ldots, K_{N}$. For the transmitting signals from the set of RAUs, the user's SINR can be expressed as follows:

$$
\gamma_{k}^{(L, N)}=\frac{\sum_{i \in D_{k}} P S_{i}\left|h_{i}\right|^{2}}{\sigma_{I z}^{2}}
$$


It is assumed that $m$ is the index and $m=\arg \max \left\{\gamma_{k}^{(L, N)}\right\}, k=1, \ldots, K_{N}$. Therefore, the maximum SINR received by the user with RAUs selection transmission is expressed as follows:

$$
\gamma_{\max }^{(L, N)}=\gamma_{m}^{(L, N)}
$$

As shown in Fig. 1, for any one user, there are three RAUs surrounding the user, so the typical value of $L$ is 3 for RAU selection. In the case of the NCT_RAUs mode, the RAU is selected from $L=3$ candidate RAUs that are near the target user. By contrast, in the CT_RAUs mode, three options are available for selecting two cooperating RAUs from three RAUs, and the combination has maximum value of SINR is selected. Thus, in the NCT_RAUS mode, one RAU is selected from three candidate RAUs to transmit signals to the served user. The user's maximum received SINR for NCT_RAUS is expressed as follows:

$$
\gamma_{\text {NCT_RAUS }}=\gamma_{\max }^{(3,1)}=\max \left\{\frac{P S_{1}\left|h_{1}\right|^{2}}{\sigma_{I z}^{2}}, \frac{P S_{2}\left|h_{2}\right|^{2}}{\sigma_{I z}^{2}}, \frac{P S_{3}\left|h_{3}\right|^{2}}{\sigma_{I z}^{2}}\right\}
$$

The user's rate with the unit bandwidth, that is, spectral efficiency, can be expressed as follows:

$$
R_{\text {NCT_RAUS }}=\log _{2}\left(1+\gamma_{\text {NCT_RAUS }}\right)
$$

In the CT_RAUS mode, two RAUs need to be selected for cooperative transmission from three RAUs, and the maximum SINR of the corresponding user is given as follows:

$$
\gamma_{\text {CT_RAUS }}=\gamma_{\max }^{(3,2)}=\max \left\{\frac{P\left(S_{1}\left|h_{1}\right|^{2}+S_{2}\left|h_{2}\right|^{2}\right)}{\sigma_{I z}^{2}}, \frac{P\left(S_{2}\left|h_{2}\right|^{2}+S_{3}\left|h_{3}\right|^{2}\right)}{\sigma_{I z}^{2}}, \frac{P\left(S_{1}\left|h_{1}\right|^{2}+S_{3}\left|h_{3}\right|^{2}\right)}{\sigma_{I z}^{2}}\right\}
$$

The user's spectral efficiency can be expressed as

$$
R_{\text {CT_RAUS }}=\log _{2}\left(1+\gamma_{\text {CT_RAUS }}\right)
$$

\section{Performance Analysis for User's SINR and Ergodic Rate}

In this section, the CDF and ergodic rate suitable for any statistical wireless channel are deduced. In addition, for a Rayleigh fading channel, the closed-form expression of CDF for SINR is given. The SINR received by the user from the $l$-th RAU follows independent exponential distribution with mean $P S_{I} / \sigma_{I z}^{2}$, and the probability density function (PDF) of the user's SINR is given as follows:

$$
f_{\gamma_{l}}(\gamma)=\frac{\sigma_{I z}^{2}}{P S_{l}} \exp \left(-\frac{\sigma_{I I}^{2}}{P S_{l}} \gamma\right), \quad \gamma \geq 0
$$




\subsection{Performance Analysis without RAU Selection}

First, consider NCT mode; when RAU1 transmits a signal to the served user, according to (10), the PDF of the user's SINR is

$$
f_{\gamma_{1}}(\gamma)=\frac{\sigma_{I z}^{2}}{P S_{1}} \exp \left(-\frac{\sigma_{I z}^{2}}{P S_{1}} \gamma\right), \quad \gamma \geq 0
$$

The CDF of the user's SINR can be obtained as

$$
F_{\gamma_{1}(\gamma)}=\int_{0}^{\gamma} f_{\mathrm{NCT}_{-} \gamma_{1}}(x) d x=1-\exp \left(-\frac{\sigma_{I z}^{2}}{P S_{1}} \gamma\right)
$$

The user's ergodic rate can be determined as follows [32]:

$$
\mathbb{E}\left[R_{N C T}\right]=\int_{0}^{\infty} \log (1+\gamma) f_{\gamma_{1}}(\gamma) d \gamma=\frac{1}{\ln 2} \exp \left(\frac{\sigma_{I z}^{2}}{P S_{1}}\right) \operatorname{Ei}\left(\frac{\sigma_{I z}^{2}}{P S_{1}}\right)
$$

where $\operatorname{Ei}(x)=\int_{x}^{\infty} \frac{\exp (-t)}{t} d t$ is the exponential integral function.

In the CT mode, when RAU1 and RAU2 cooperate to transmit signals to the user, the CDF and PDF are derived as follows. In the following formulas, superscript 1 represents $\lambda_{i} \neq \lambda_{j}$, and superscript 2 represents $\lambda_{i}=\lambda_{j}$. When $\lambda_{i} \neq \lambda_{j}$, the CDF of the user's SINR is derived as follows:

$$
\begin{aligned}
F_{\gamma_{1}+\gamma_{2}(\gamma)}^{1} & =P\left(\gamma_{1}+\gamma_{2} \leq \gamma\right) \\
& =\iint_{\gamma_{1}+\gamma_{1} \leq \gamma} \frac{\sigma_{I z}^{2}}{P S_{1}} \exp \left(-\frac{\sigma_{I z}^{2}}{P S_{1}} \gamma_{1}\right) \frac{\sigma_{I z}^{2}}{P S_{2}} \exp \left(-\frac{\sigma_{I z}^{2}}{P S_{2}} \gamma_{2}\right) d \gamma_{1} d \gamma_{2} \\
& =1-\frac{S_{1}}{S_{1}-S_{2}} \exp \left(-\frac{\sigma_{I z}^{2}}{P S_{1}} \gamma\right)-\frac{S_{2}}{S_{2}-S_{1}} \exp \left(-\frac{\sigma_{I z}^{2}}{P S_{2}} \gamma\right)
\end{aligned}
$$

The PDF is obtained using the CDF as follows:

$$
f_{\gamma_{1}+\gamma_{2}(\gamma)}^{1}=\frac{d F_{\gamma_{1}+\gamma_{2}(\gamma)}^{1}}{d \gamma}=\frac{\sigma_{I z}^{2}}{P\left(S_{1}-S_{2}\right)}\left[\exp \left(-\frac{\sigma_{I z}^{2}}{P S_{1}} \gamma\right)-\exp \left(-\frac{\sigma_{I z}^{2}}{P S_{2}} \gamma\right)\right]
$$

The user's ergodic rate can be expressed as follows:

$$
\begin{aligned}
\mathbb{E}\left[R_{\mathrm{CT}}^{1}\right] & =\int_{0}^{\infty} \log _{2}(1+\gamma) f_{\gamma_{1}+\gamma_{2}}^{1}(\gamma) d \gamma \\
& =\sum_{l=1}^{2} \frac{1}{\ln 2}\left(\prod_{t=1, t \neq l}^{2} \frac{S_{l}}{S_{l}-S_{t}}\right) \exp \left(\frac{\sigma_{I z}^{2}}{P S_{l}}\right) \operatorname{Ei}\left(\frac{\sigma_{I z}^{2}}{P S_{l}}\right)
\end{aligned}
$$


When $\lambda_{1}=\lambda_{2}$, let $\lambda_{1}=\lambda_{2}=\lambda$; then, the PDF of the user's SINR can be obtained as follows:

$$
f_{\gamma_{1}+\gamma_{2}}^{2}(\gamma)=\int_{-\infty}^{\infty} f_{\gamma}(x) f_{\gamma}(\gamma-x) d x=\left(\frac{\sigma_{I z}^{2}}{P S}\right)^{2} \gamma \exp \left(-\frac{\sigma_{I z}^{2}}{P S} \gamma\right)
$$

In this case, the CDF of the user's SINR is

$$
F_{\gamma_{1}+\gamma_{2}(\gamma)}^{2}=1-\left(1+\frac{\sigma_{I z}^{2}}{P S} \gamma\right) \exp \left(-\frac{\sigma_{I z}^{2}}{P S} \gamma\right)
$$

The user's ergodic rate is derived as follows:

$$
\begin{aligned}
\mathbb{E}\left[R_{\mathrm{CT}}^{2}\right] & =\int_{0}^{\infty}\left[\log _{2}(1+\gamma)\right]\left(\frac{\sigma_{I z}^{2}}{P S}\right)^{2} \gamma \exp \left(-\frac{\sigma_{I z}^{2}}{P S} \gamma\right) d \gamma \\
& =\frac{P S}{\sigma_{I z}^{2} \ln 2}\left[\left(\frac{\sigma_{I z}^{2}}{P S}-1\right) \exp \left(\frac{\sigma_{I z}^{2}}{P S}\right)\left(\exp \left(-\frac{\sigma_{I z}^{2}}{P S}\right)-\frac{\sigma_{I z}^{2}}{P S} \operatorname{Ei}\left(\frac{\sigma_{I z}^{2}}{P S}\right)\right)+1\right]
\end{aligned}
$$

\subsection{Performance Analysis with RAU Selection}

In the NCT_RAUS mode, the RAU with the best channel condition to the user is selected as the transmitter, and the CDFs of SINR for three RAUs are denoted as $F_{\gamma_{1}(\gamma)}, F_{\gamma_{2}(\gamma)}, F_{\gamma_{3}(\gamma)}$. When $\lambda_{1} \neq \lambda_{2} \neq \lambda_{3}$, the CDF of the user's SINR for NCT_RAUS is as follows:

$$
F_{\text {NCT_RAUS }(\gamma)}^{1}=F_{\gamma_{1}(\gamma)} F_{\gamma_{2}(\gamma)} F_{\gamma_{3}(\gamma)}=\prod_{l=1}^{3}\left[1-\exp \left(-\frac{\sigma_{I z}^{2}}{P S_{l}} \gamma\right)\right]
$$

The corresponding PDF of the user's SINR can be expressed as follows:

$$
\begin{aligned}
f_{\text {NCT_RAUS }(\gamma)=}^{1}= & \sum_{l=1}^{3} \frac{\sigma_{I z}^{2}}{P S_{l}} \exp \left(-\frac{\sigma_{I z}^{2}}{P S_{l}} \gamma\right)-\frac{\sigma_{I t}^{2}}{P} \sum_{l=1}^{3}\left(\sum_{t=1, t \neq l}^{3} \frac{1}{S_{t}}\right) \exp \left[-\frac{\sigma_{I z}^{2}}{P}\left(\sum_{t=1, t \neq l}^{3} \frac{1}{S_{t}}\right) \gamma\right] \\
& +\frac{\sigma_{I t}^{2}}{P}\left(\sum_{l=1}^{3} \frac{1}{S_{l}}\right) \exp \left[-\frac{\sigma_{I z}^{2}}{P}\left(\sum_{l=1}^{3} \frac{1}{S_{l}}\right) \gamma\right]
\end{aligned}
$$

When $\lambda_{1}=\lambda_{2}=\lambda_{3}$, the CDF of the user's SINR for NCT_RAUS is as follows:

$$
F_{\text {NCT_RAUS }(\gamma)}^{2}=\left[F_{\gamma_{1}(\gamma)}\right]^{3}=\left[1-\exp \left(-\frac{\sigma_{I}^{2}}{P S} \gamma\right)\right]^{3}
$$

In the CT_RAUS mode, when $\lambda_{1} \neq \lambda_{2} \neq \lambda_{3}$, the CDF of the user's SINR is given as follows:

$$
F_{\text {CT_RAUS }(\gamma)}^{1}=F_{\gamma_{1}+\gamma_{2}(\gamma)} F_{\gamma_{2}+\gamma_{3}(\gamma)} F_{\gamma_{1}+\gamma_{3}(\gamma)}
$$


When $\lambda_{1}=\lambda_{2}=\lambda_{3}$, the CDF of the user's SINR for CT_RAUS is derived as follows:

$$
F_{\text {CT_RAUS }(\gamma)}^{2}=\left[1-\left(1+\frac{\sigma_{I I}^{2}}{P S} \gamma\right) \exp \left(-\frac{\sigma_{I z}^{2}}{P S} \gamma\right)\right]^{3}
$$

The derivative of CDF yields the corresponding PDF, and the ergodic rate can be obtained easily thereafter.

\section{Spectral and Energy Efficiency Tradeoff}

$\mathrm{SE}$ is the main performance index for designing and optimizing wireless communication networks. It indicates the efficiency of limited spectrum resources but does not indicate the EE. Therefore, it is necessary to find suitable indicators to represent the EE of the system. The EE of a communication network can be measured in units of bit per joule [33]-[35], which denotes the maximum number of transmitted bits per joule of energy consumed. The actual power consumption model should include the transmission power, efficiency of the power amplifier (PA), circuit power consumption, and backhaul overheads, etc. In this section, the power consumption model of a single DAS cell is first introduced, and then, the closed-form tradeoff relationship between SE and EE is derived.

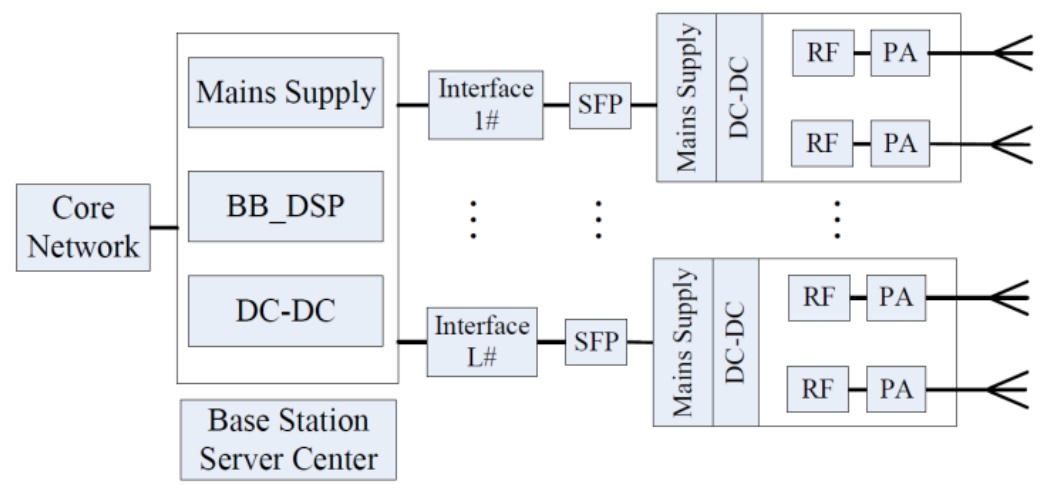

Fig. 2. DAS power model

\subsection{DAS Power Consumption Model}

Consider the power model of a single DAS cell, as shown in Fig. 2, where the number of RAUs in the cell is seven. The PA and RF are in the same geographical location as the corresponding RAU, whereas the baseband (BB) processing unit is in the central unit. Compared to the transceiver of the general base station, the transceiver of a RAU does not require a feeder cable, so there is no feedback loss. The total power consumption during downlink can be expressed as follows [36]:

$$
P_{t d}=L T \zeta P+L P_{s c}+L P_{b h}+K P_{c r}
$$

where $L$ is the number of cooperative RAUs, $T$ represents the number of antennas on each RAU, $\zeta \in[0,1]$ is the power amplifier efficiency, and $P$ is the transmission output power of the power amplifier. $P_{s c}$ is the static circuit power consumption of each RAU. $P_{b h}$ denotes the power consumption of a backhaul link. $K$ is the number of served users, and $P_{c r}$ is the power 
required for reception by a user in the downlink. The power consumption model for a single backhaul link is as follows [37]:

$$
P_{b h}=\left[\omega P_{C U}+(1-\omega)\left(\tau_{\mathrm{s}} / \tau_{\max }\right) P_{C U}\right] / N_{\mathrm{RAU}}+P_{d l}
$$

where $P_{C U}$ is the maximum power consumption of the central unit when the backhaul information is processed, and $\omega \in[0,1]$ is a weighting factor. $\tau_{\mathrm{s}}$ and $\tau_{\max }$ represent the actual traffic load and the total maximum traffic load, respectively. $N_{\mathrm{RAU}}$ is the maximum number of RAUs that the central unit can afford, $P_{d l}$ is the power consumption of each interface.

\section{2 SE-EE Tradeoff Formulation}

For the sake of simplicity, in this section, only the CT mode is studied, and the analysis results can be easily extended to the other transmission modes. When $L$ RAUs in cell-0 simultaneously transmit signals to the user, the system SE can be expressed as follows:

$$
C_{L}=\log _{2}\left(1+\frac{\sum_{l=1}^{L} S_{l} P\left|h_{l}\right|^{2}}{\sigma_{I z}^{2}}\right)
$$

The transmission output power $P$ of the power amplifier can be obtained as follows:

$$
P=\frac{\sigma_{I z}^{2}\left(2^{C_{L}}-1\right)}{\sum_{l=1}^{L} S_{l}\left|h_{l}\right|^{2}}
$$

The system capacity $C=B C_{L}$, where $B$ is the system bandwidth, and system EE is expressed as follows:

$$
C_{J}=\frac{C}{P_{t d}}=\frac{B C_{L}}{L T \zeta P+\omega P_{C U} / N_{\mathrm{RAU}}+P_{s c}+P_{d l}+(1-\omega) \tau_{\mathrm{s}} P_{C U} L /\left(\tau_{\max } N_{\mathrm{RAU}}\right)+K P_{c r}}
$$

By substituting (28) into (29), the tradeoff relationship between SE and EE can be formulated as follows:

$$
C_{J}=\frac{B C_{L}}{L T \zeta \sigma_{I z}^{2}\left(2^{C_{L}}-1\right) / \sum_{l=1}^{L} S_{l}\left|h_{l}\right|^{2}+(1-\omega) \tau_{\mathrm{s}} P_{C U} L /\left(\tau_{\max } N_{\mathrm{RAU}}\right)+L\left(\omega P_{C U} / N_{\mathrm{RAU}}+P_{d l}+P_{\mathrm{sc}}\right)+K P_{c r}}
$$

\section{Simulation Results}

Consider a multi-cell DAS, as shown in Fig. 1, where RAU1 in cell-0 is located at the center of the cell, and the remaining six RAUs are distributed uniformly within the cell. The polar coordinates of RAU1-RAU7 are RAU1 $(0,0)$, RAU2 $(d, 0)$, RAU3 $(d, \pi / 3)$, RAU4 ( , 
$2 \pi / 3$ ), RAU5 ( $d, \pi)$, RAU6 ( $d, 4 \pi / 3$ ), and RAU7 $(d, 5 \pi / 3)$, where $d=500 \mathrm{~m}$ [38]. A similar RAU deployment structure is used in the other cells as well. It is assumed that the cell radius is $750 \mathrm{~m}$, and the path loss exponent $\alpha$ is 3.76. The average transmission power of each active RAU $P=43 \mathrm{dBm}$, and the average noise power $\sigma_{z}^{2}=-104 \mathrm{dBm}$. It is assumed that both the transmitter and the receiver know the perfect CSI. The worst case is considered in this paper, which means that the RAUs of the six cells surrounding cell- 0 are all active, that is, $L_{c}=7, \forall c$.

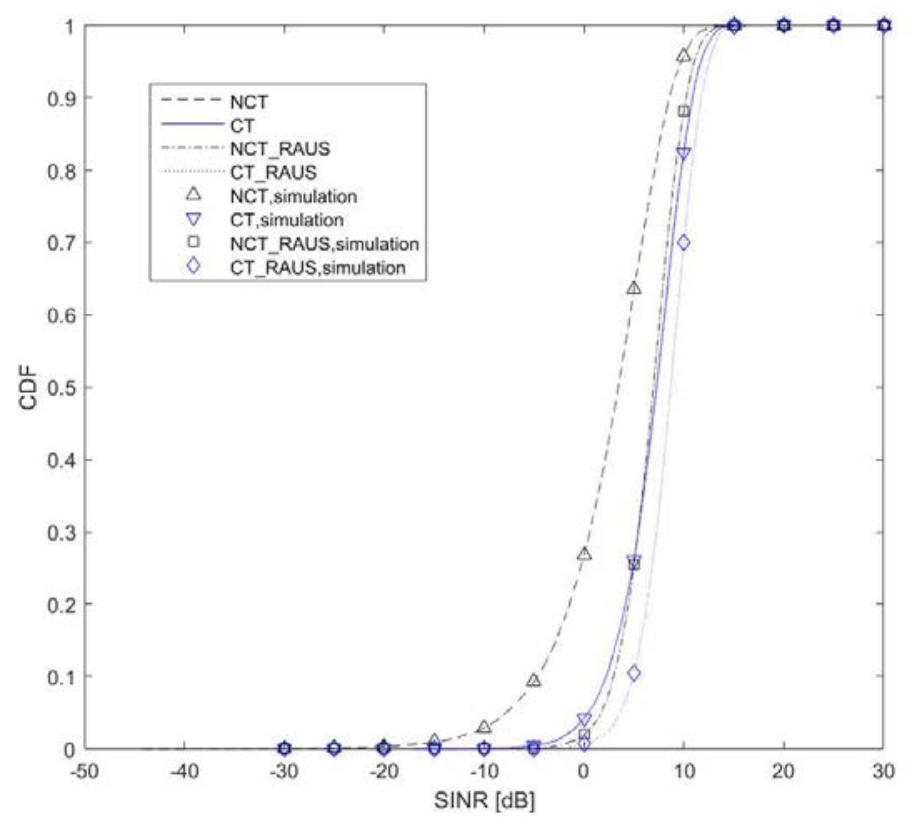

Fig. 3. CDF of user's SINR when the user is located at the center of RAU1, RAU2, and RAU3

The CDF curves of the user's SINR are shown in Fig. 3. The four transmission modes of NCT, CT, NCT_RAUS, and CT_RAUS are considered, and the user is at the center point A of RAU1, RAU2, RAU3 in cell-0. In this case, the average power received by the user from the three RAUs is the same. As can be seen from Fig. 3, regardless of whether RAU selection is performed, the SINR performance in the CT mode is better than that in the NCT mode, which fully demonstrates that the CT mode can boost SINR performance. From (2) and (3), we have $\gamma_{\mathrm{CT}}>\gamma_{\mathrm{NCT}}$. The simulation results in Fig. 3 verify the correctness of the derivation. Furthermore, the SINR performances in both the NCT and the CT modes with RAU selection move to the right because RAU selection can enhance SINR performance. The user's SINR performance is the best in the CT_RAUS mode because this mode makes full use of the performance gains due to cooperation and selection. The CDF of SINR by simulation is given as well, where the simulation values of SINR were obtained through 10,000 Monte Carlo simulations. Fig. 3 shows that all analytical curves are consistent with the Monte Carlo simulation results.

Fig. 4 shows the CDF curves of the user's SINR in the NCT, CT, NCT_RAUS, and CT_RAUS transmission modes, where the user is located within the right-angled triangle OAD, as shown in Fig. 1. The average received power values of the user from RAU1, RAU2, and RAU3 are different from each other. The user is always served by RAU1 for NCT, while the probability that RAU1 services the user in the NCT_RAUS mode decreases. RAU1 and 
RAU2 cooperatively service the user in the CT mode, while RAU1 and RAU2 can serve the user with greater probability in the CT_RAUS mode. The SNR distribution of the user is closely related to the user's location. Similar to Fig. 3, all analytical curves are consistent with the results of Monte Carlo simulation.

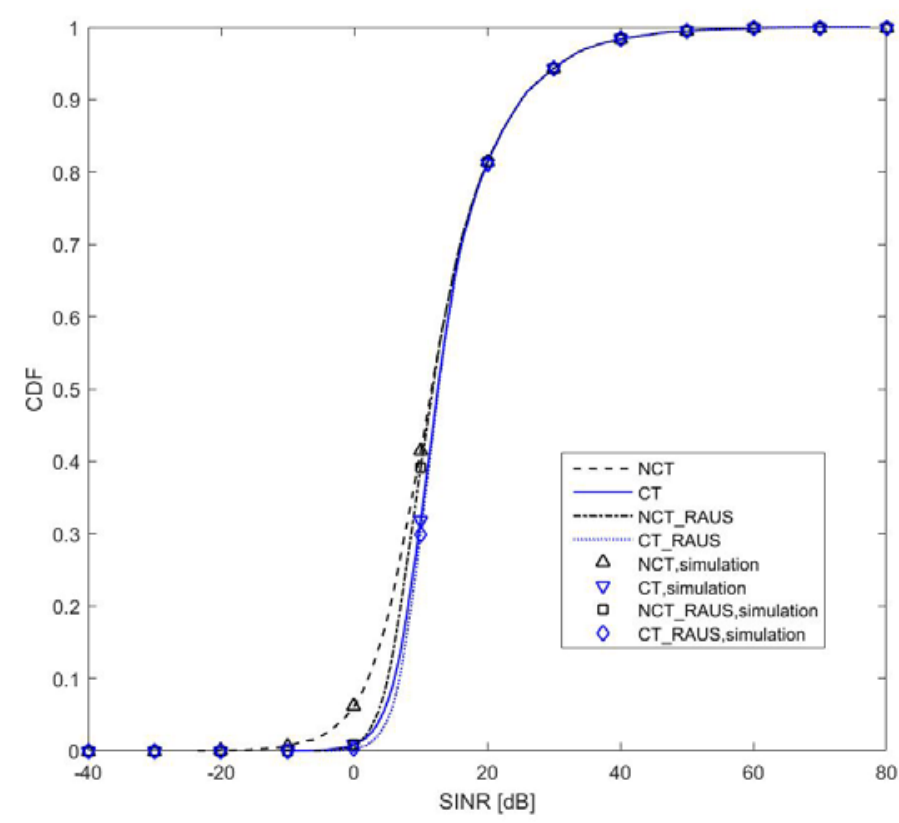

Fig. 4. CDF of user's SINR, where the user is located within the right-angled triangle OAD

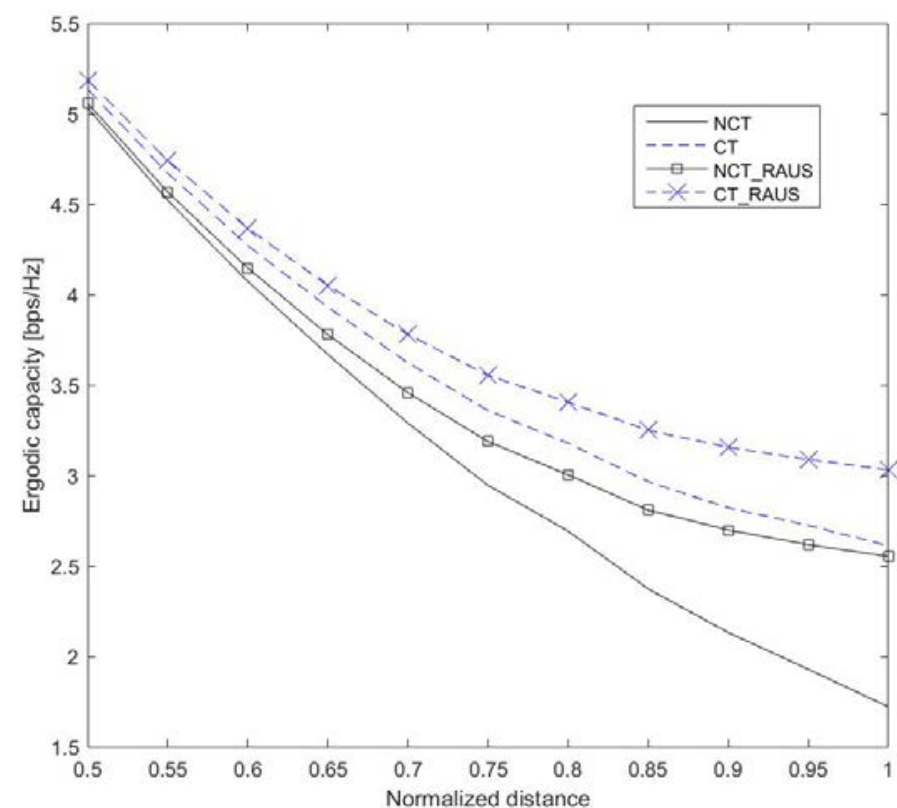

Fig. 5. Ergodic capacity versus normalized distance in different transmission modes 
Fig. 5 shows how the ergodic capacity varies with the user's distance in different transmission modes. The range of the reference distance is the line segment OA shown in Fig. 1. The normalized distance of point $\mathrm{O}$ is 0 , and the normalized distance of point $\mathrm{A}$ is 1 . When the user moves from $\mathrm{O}$ to $\mathrm{A}$, the distance from the user to RAU1 increases, and the ergodic capacity of the system decreases in all four transmission modes. At the same user location, the ergodic capacity of the system is better in the CT mode than in the NCT mode. As the distance increases, the ergodic capacity decreases dramatically in the NCT mode. By contrast, when CT or RAU selection is used, the ergodic capacity decreases gradually. This is because CT and RAU selection are effective for improving the ergodic capacity of the system.

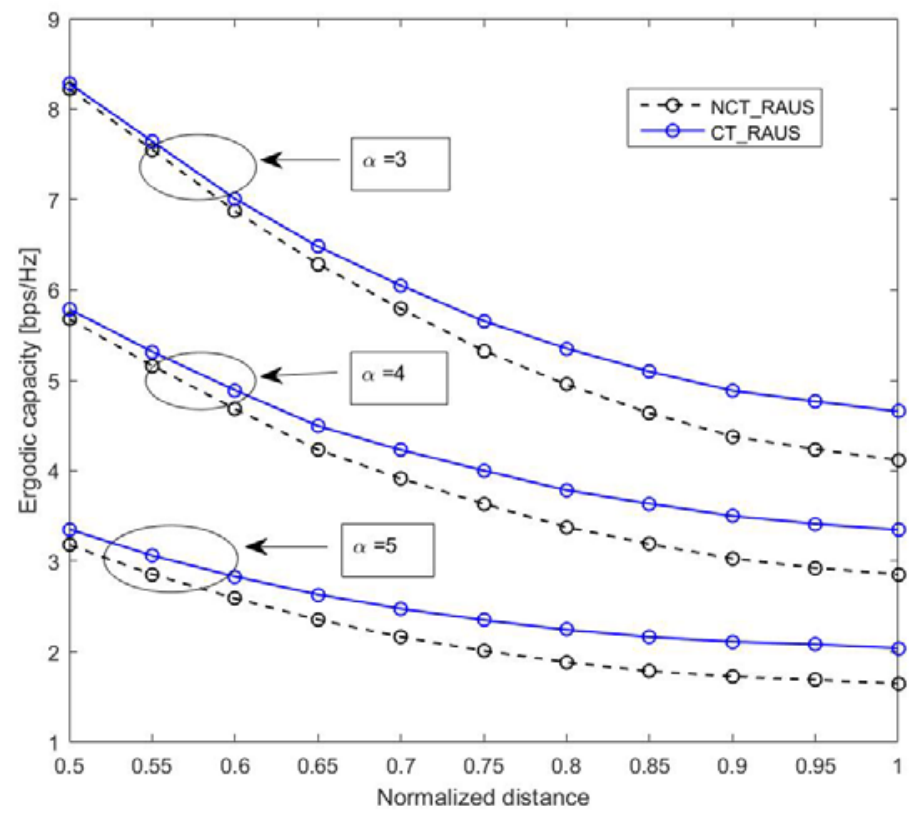

Fig. 6. Ergodic capacity versus normalized distance for different path loss exponents

The relationships between the ergodic capacity and the normalized distance in case of the NCT_RAUS and the CT_RAUS modes are shown in Fig. 6, when the path loss exponents are 3, 4 and 5. As the path loss exponent increases, the ergodic capacity in the NCT_RAUS and CT_RAUS modes decreases. This is because as the path loss exponent increases, power attenuation from the RAU to the user intensifies, and the corresponding SINR decreases. For the same path loss exponent and location, the ergodic capacity in the CT_RAUS mode is always larger than that in the NCT_RAUS mode. With increasing normalized distance, the ergodic capacity gap between the CT_RAUS and the NCT_RAUS modes grows. Similar to the results in Fig. 5, RAU cooperation and selection can increase system capacity.

The simulation results of SE and EE are as follows. The path loss exponent $\alpha=3.76$. The maximum transmission power per active RAU $P_{\max }$ is $45 \mathrm{dBm}$, and the output power at the RF $P \in\left[0, P_{\max }\right]$. The number of antennas $T$ on each RAU is 1 . It is assumed that all RAUs have the same antenna and amplifier, and the power amplifier efficiency $\zeta$ is 2.8. The static power consumption $P_{s c}$ is $59.2 \mathrm{~W}$, and power consumption of the user $P_{c r}$ is $0.1 \mathrm{~W}$. The maximum power consumption of the central unit $P_{C U}$ is $300 \mathrm{~W}$, and the weighting factor $\omega$ is 0.5 . The maximum number of RAUs $N_{\text {RAU }}$ is 24 . The power consumption at each interface $P_{d l}$ is $1 \mathrm{~W}$. 
The backhaul link includes both uplink and downlink, and for simplicity, it is assumed that $\tau_{\mathrm{s}} / \tau_{\max }$ is $0.5[36]$.

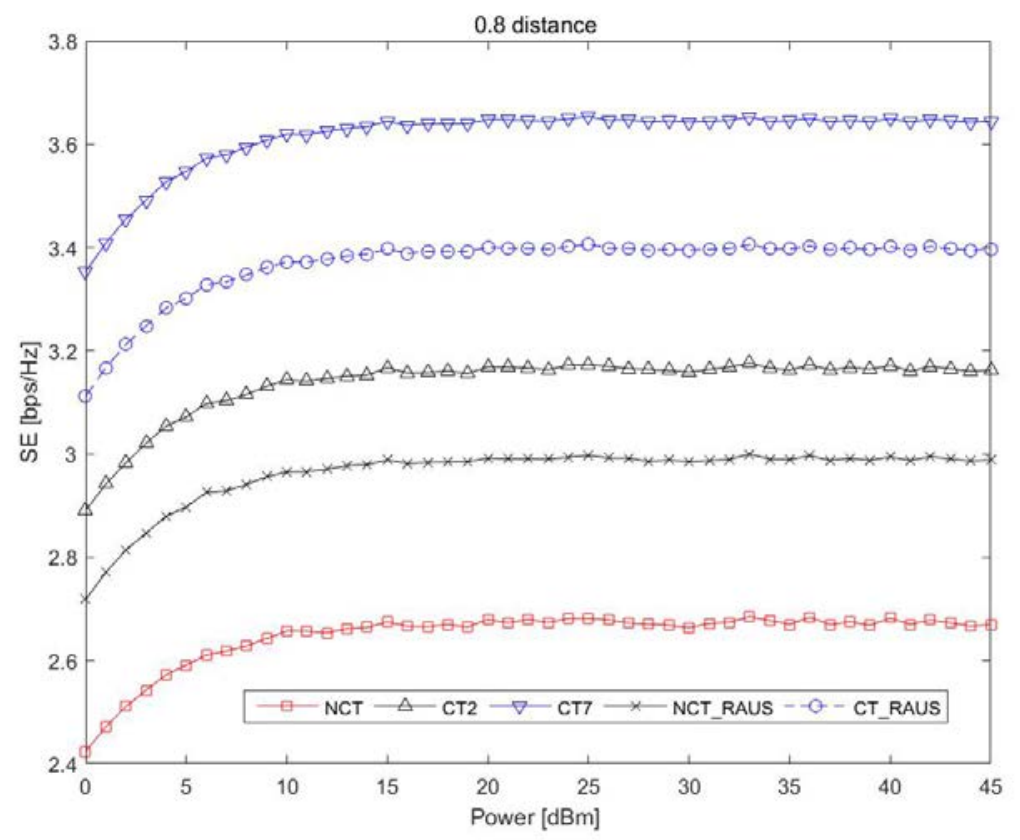

Fig. 7. System SE versus actual transmission power when normalized distance is 0.8

Fig. 7 shows how system SE varies with the actual transmission power when the normalized distance is 0.8 . The system SE in the four transmission modes increases as the transmission power increases, and there is no crossing point. When seven RAUs fully cooperate for transmission, that is, CT7, the system SE is the highest. This is because CT7 fully exploits the total diversity gain. The system SE in the CT mode is better than that in the NCT mode. The system SE increases from low to high in the following order: NCT, NCT_RAUS, CT2, CT_RAUS, and CT7. Fig. 7 provides useful insights from the viewpoint of designing a network with SE as the priority.

A plot of system EE versus actual transmission power is shown in Fig. 8, and the normalized distance is 0.8. The system EE in each of the transmission modes increases with increasing transmission power until the maximum EE is reached; thereafter, the system EE decreases with increasing transmission power. System EE is determined by the system capacity and the corresponding total power consumption. For a given power level, the NCT_RAUS mode shows the highest EE value.

The tradeoff relationship between system SE and EE is plotted in Fig. 9, where the user's normalized distance is 0.8 . EE first increases with SE. After reaching a maximum value, EE begins to decrease. Owing to the influence of interference, as the power level increases, SE first increases and then shows almost no change. Notably, for different transmission modes, the maximum EE values are different, and the corresponding SE values are different as well. In the NCT_RAUS mode, the maximum EE value is about 0.042 bit/joule, and the corresponding SE value is $3 \mathrm{bps} / \mathrm{Hz}$. In the NCT mode, the maximum EE value is about 0.038 bit/joule, and the corresponding SE value is $2.65 \mathrm{bps} / \mathrm{Hz}$. In network design in the future, system SE and EE should be considered comprehensively. For different optimization objectives, we must carefully select a reasonable transmission mode. 


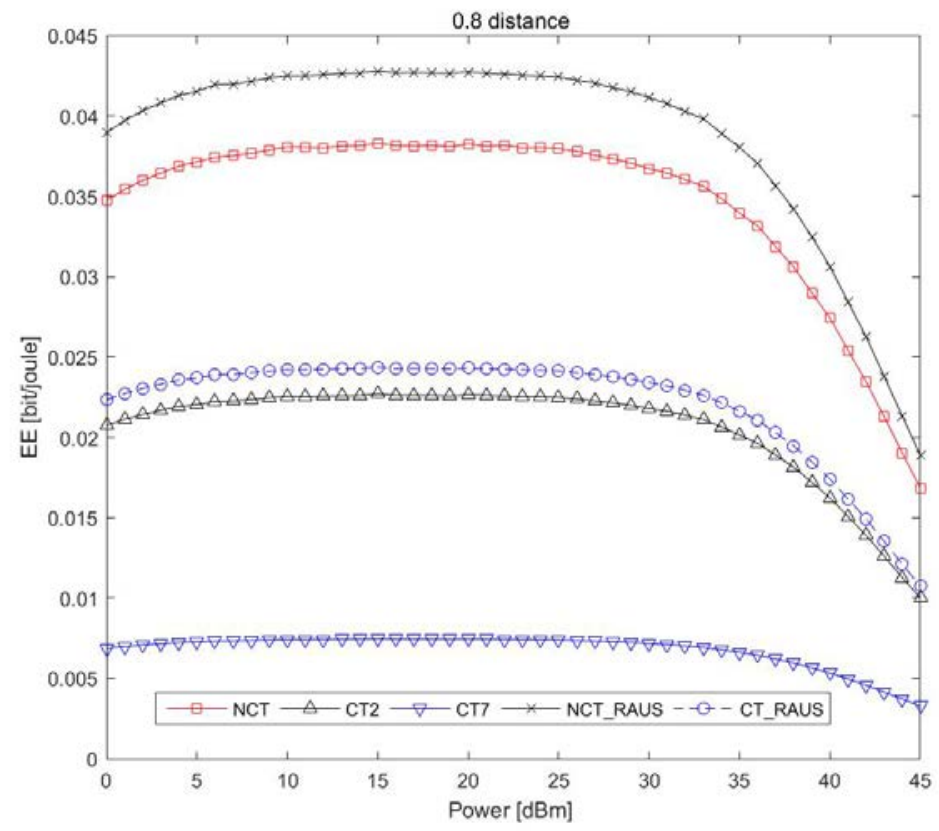

Fig. 8. System EE versus actual transmission power when normalized distance is 0.8

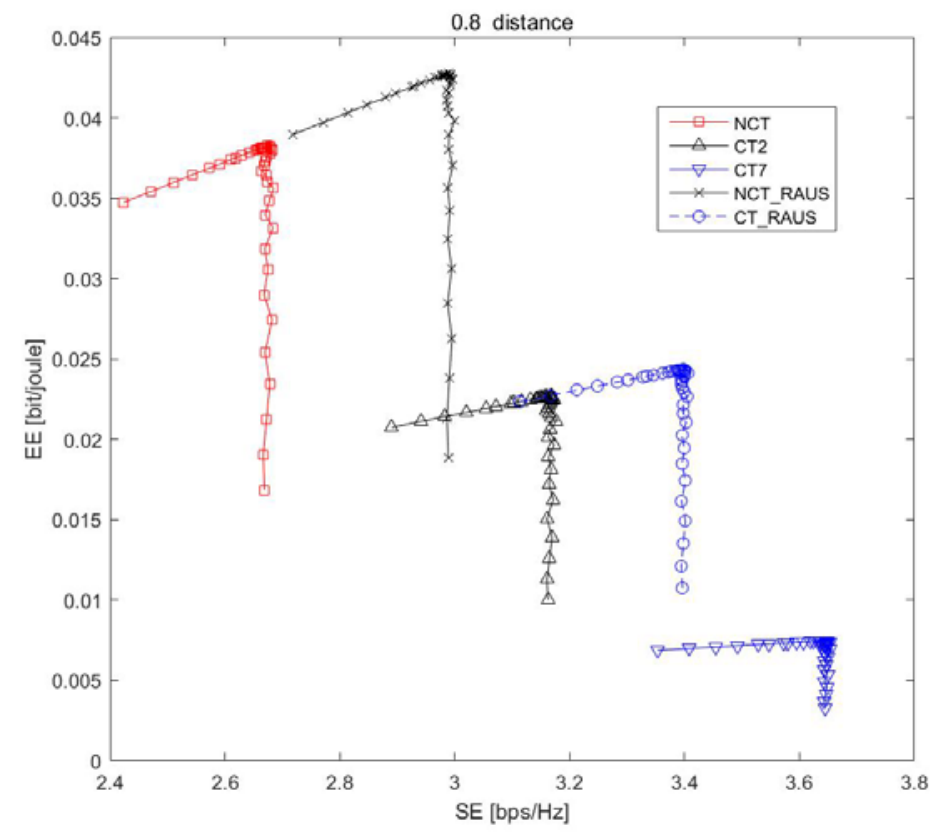

Fig. 9. Tradeoff curves between SE and EE when normalized distance is 0.8 


\section{Conclusion}

In this work, the performance of DAS is investigated considering RAU selection and cooperation, including user's SINR, ergodic capacity, and system SE and EE. When active RAUs transmit signals to the user, the other RAUs in this cell that do not transmit the signals are in a closed state. Through RAU selection and cooperation, the four transmission modes, namely, NCT, CT, NCT_RAUS, and CT_RAUS, are formulated. By using the probability theory, the CDF of SINR and the ergodic capacity are deduced theoretically, and the corresponding closed-form expressions are developed. With the introduction of a detailed power consumption model of DAS, the system EE is studied. The expression of EE is formulated, and the tradeoff relationship between SE and EE is deduced as well. The simulation results show that the CDF curves of the user's SINR and the ergodic capacity obtained by theoretical derivation agree well with the results of Monte Carlo simulation. From the simulation results, it can be seen that system EE is constrained by system capacity and the actual power consumption mode. This provides a scientific guideline for optimizing the design of future networks.

\section{References}

[1] G. J. Foschini and M. Gans, "On limits of wireless communications in a fading environment when using multiple antennas,” Wireless Personal Communication, vol. 6, no. 3, pp. 311-335, March, 1998. Article (CrossRef Link)

[2] H. Lee, B. Lee and I. Lee, "Iterative detection and decoding with an improved V-BLAST for MIMOOFDM systems," IEEE Journal on Selected Areas in Communications, vol. 24, no. 3, pp. 504-513, March, 2006. Article (CrossRef Link)

[3] I. Lee, A. M. Chan and C. E. W. Sundberg, "Space-time bit-interleaved coded modulation for OFDM systems," IEEE Transactions on Signal Processing, vol. 52, no. 3, pp. 820-825, March, 2004. Article (CrossRef Link)

[4] M. V. Clark, T. M. Willis, L. J. Greenstein, A. J. Rustako, V. Erceg and R. S. Roman, "Distributed versus centralized antenna arrays in broadband wireless networks," in Proc. of IEEE Vehicular Technology Conf., pp. 33-37, May 6-9, 2001. Article (CrossRef Link)

[5] W. Roh and A. Paulraj, "Outage performance of the distributed antenna systems in a composite fading channel," in Proc. of IEEE Vehicular Technology Conf., pp. 1520-1524, September 24-28, 2002. Article (CrossRef Link)

[6] 3GPP-LTE, "Technical specification group radio access network: Evolved universal terrestrial radio access (E-UTRA): further advancements for E-UTRA physical layer aspects (Release 9)," 3GPP TR36.814, March, 2010. Article (CrossRef Link)

[7] W. Peng and F. Adachi, "Multi-user downlink transmit beamforming for the broadband single-carrier distributed antenna network," in Proc. of IEEE Vehicular Technology Conf., pp. 1-5, September 5-8, 2011. Article (CrossRef Link)

[8] F. Adachi, K. Takeda, T. Yamamoto and R. Matsukawa, "Gigabit distributed antenna network and its related wireless techniques," in Proc. of 7th Int. Wireless Communications and Mobile Computing Conf., pp. 1550-1556, July 4-8, 2011. Article (CrossRef Link)

[9] F. Yuan, S. Jin, K. K. Wong, J. Zhao and H. Zhu, "Wireless information and power transfer design for energy cooperation distributed antenna systems," IEEE Access, vol. 5, pp. 8094-8105, March, 2017. Article (CrossRef Link)

[10] H. Kim, E. Park, H. Park and I. Lee, "Beamforming and power allocation designs for energy efficiency maximization in MISO distributed antenna systems,” IEEE Communication Letters, vol. 17, no. 11, pp. 2100-2103, November, 2013. Article (CrossRef Link) 
[11] X. Wang, P. Zhu and M. Chen, "Antenna location design for generalized distributed antenna systems,” IEEE Communication Letters, vol. 13, no. 5, pp. 315-317, May, 2009. Article (CrossRef Link)

[12] E. Park and I. Lee, "Antenna placement for downlink distributed antenna systems with selection transmission,” in Proc. of IEEE Vehicular Technology Conf., pp. 1-5, May15-18, 2011. Article (CrossRef Link)

[13] L. Han, T. Liu, S. Shao, Y. Shen and Y. Tang, "Optimal antenna location for circular layout distributed antenna systems with uplink selected maximal ratio combining," in Proc. of Int. Conf. on Communications, Circuits and Systems, pp. 137-140. November 15-17, 2013. Article (CrossRef Link)

[14] L. Dai, S. Zhou and Y. Yao, "Capacity analysis in CDMA distributed antenna systems," IEEE Transactions on Wireless Communications, vol. 4, no. 6, pp. 2613-2620, November, 2005. Article (CrossRef Link)

[15] L. Dai, "An uplink capacity analysis of the distributed antenna system (DAS): from cellular DAS to DAS with virtual cells," IEEE Transactions on Wireless Communications, vol. 13, no. 5, pp. 2717-2731, May, 2014. Article (CrossRef Link)

[16] L. Xiao, L. Dai, H. Zhuang, S. Zhou and Y. Yao, "Information-theoretic capacity analysis in MIMO distributed antenna systems," in Proc. of IEEE Vehicular Technology Conf., pp. 779-782, April 22-25, 2003. Article (CrossRef Link)

[17] R. Hasegawa, M. Shirakabe, R. Esmailzadeh and M. Nakagawa, "Downlink performance of a CDMA system with distributed base station," in Proc. of IEEE Vehicular Technology Conf., pp. 882-886, October 6-9, 2003. Article (CrossRef Link)

[18] H. Kim, S. R. Lee and I. Lee, "Transmission schemes based on sum rate analysis in distributed antenna systems," IEEE Transactions on Wireless Communications, vol. 11, no. 3, pp. 1201-1209, March, 2012. Article (CrossRef Link)

[19] W. Choi and J. G. Andrews, "Downlink performance and capacity of distributed antenna systems in a multicell environment," IEEE Transactions on Wireless Communications, vol. 6, no. 1, pp. 69-73, January, 2007. Article (CrossRef Link)

[20] C. He, B. Sheng, P. Zhu and X. You, "Energy efficiency and spectral efficiency tradeoff in downlink distributed antenna systems," IEEE Wireless Communication Letters, vol. 1, no. 3, pp. 153-156, June, 2012. Article (CrossRef Link)

[21] C. He, B. Sheng, D. Wang, P. Zhu and X. You, "Energy efficiency comparison between distributed MIMO and co-located MIMO systems," Int. Journal of Communication Systems, vol. 27, no. 1, pp. 81-94, January, 2014. Article (CrossRef Link)

[22] Q. Pang, X. Wang, W. Wan, Y. Zhao and X. Gu, "Coordinated beamforming for energy efficient transmission in distributed antenna systems," in Proc. of IEEE Personal, Indoor, and Mobile Radio Communication, pp. 1639-1643, August 30-September 2, 2015. Article (CrossRef Link)

[23] M. L. Tham, S. F. Chien, D. W. Holtby and S. Alimov, "Energy-efficient power allocation for distributed antenna systems with proportional fairness," IEEE Transactions on Green Communications and Networking, vol. 1, no. 2, pp. 145-157, June, 2017. Article (CrossRef Link)

[24] W. Xu and M. Lin, "Downlink capacity analysis of distributed antenna systems with imperfect channel state information," KSII Transactions on Internet and Information Systems, vol. 11, no. 1, pp. 253-271, January, 2017. Article (CrossRef Link)

[25] H. Ren, N. Liu, C. Pan and C. He, "Energy efficiency optimization for MIMO distributed antenna systems," IEEE Transactions on Vehicular Technology, vol. 66, no. 3, pp. 2276-2288, March, 2017. Article (CrossRef Link)

[26] X. Yu, H. Wang, X. Wang, G. Wang and X. Dang, "Energy-efficient power allocation scheme for distributed antenna system over composite fading channels," IEEE Access, vol. 6, pp. 18108-18116, March, 2018. Article (CrossRef Link)

[27] N. Akbar, E. Bjoernson, E. G. Larsson and N. Yang, "Downlink power control in massive MIMO networks with distributed antenna arrays," in Proc. of IEEE Int. Conf. on Communications, pp. 1-6, May 20-24, 2018. Article (CrossRef Link) 
[28] Y. Huang, M. Liu and Y. Liu, “Energy-efficient SWIPT in IoT distributed antenna systems,” IEEE Internet of Things Journal, vol. 5, no. 4, pp. 2646-2656, January, 2018. Article (CrossRef Link)

[29] C. Meng, T. Liang, W. Heng and X. Wang, "Multiple base stations cooperation: a novel clustering algorithm and its energy efficiency," Wireless Personal Communications, vol. 86, no. 2, pp. 351-365, January, 2016. Article (CrossRef Link)

[30] J. Park, J. Kim and W. Sung, "Performance of distributed MISO systems using cooperative transmission with antenna selection,” Journal of Communications and Networks, vol. 10, no. 2, pp. 163-174, June, 2008. Article (CrossRef Link)

[31] J. Park, E. Song and W. Sung, "Capacity analysis for distributed antenna systems using cooperative transmission schemes in fading channels," IEEE Transactions on Wireless Communications, vol. 8, no. 2, pp. 586-592, February, 2009. Article (CrossRef Link)

[32] A. Jeffrey and D. Zwillinger, Table of Integrals, Series, and Products, 7th edition. Academic Press, 2007. Article (CrossRef Link)

[33] S. Cui, A. Goldsmith and A. Bahai, "Energy-efficiency of MIMO and cooperative MIMO techniques in sensor networks," IEEE Journal on Selected Areas in Communications, vol. 22, no. 6, pp. 1089-1098, August, 2004. Article (CrossRef Link)

[34] G. Miao, N. Himayat, Y. Li and D. Bormann, "Energy efficient design in wireless OFDMA,” in Proc. of IEEE Int. Conf. on Communications, pp. 3307-3312, May 19-23, 2008. Article (CrossRef Link)

[35] W. Feng, Y. Chen, N. Ge and J. Lu, "Optimal energy-efficient power allocation for distributed antenna systems with imperfect CSI," IEEE Transactions on Vehicular Technology, vol. 65, no. 9, pp. 7759-7763, September, 2016. Article (CrossRef Link)

[36] O. Onireti, F. Heliot and M. A. Imran, "On the energy efficiency-spectral efficiency trade-off of distributed MIMO systems,” IEEE Transactions on Communications, vol. 61, no. 9, pp. 3741-3753, September, 2013. Article (CrossRef Link)

[37] S. Tombaz, P. Monti, K. Wang, A. Vastberg, M. Forzati and J. Zander, "Impact of backhauling power consumption on the deployment of heterogeneous mobile networks," in Proc. of IEEE Global Telecommunications Conf., pp. 1-5, December 5-9, 2011. Article (CrossRef Link)

[38] X. You, D. Wang, P. Zhu and B. Sheng, "Cell Edge Performance of Cellular Mobile Systems," IEEE Journal on Selected Areas in Communications, vol. 29, no. 6, pp. 1139-1150, June, 2011. Article (CrossRef Link) 


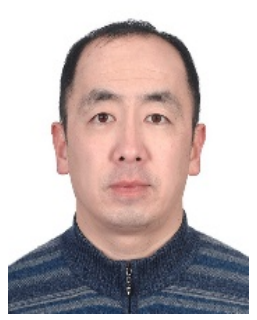

Gang Wang received the B.S. degree in electrical engineering and the M.S. degree in circuit and system from Southeast University, Nanjing, in 2000 and 2003, respectively, where he is currently pursuing the Ph.D. degree in communications and information systems. He is also a teacher at the National Mobile Communications Research Laboratory, Southeast University. His research interests include green communications, energy harvesting, and wireless communication theory.

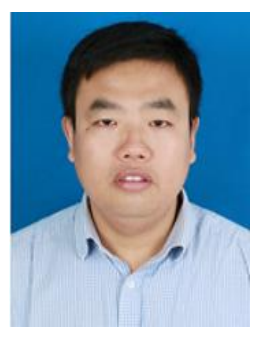

Chao Meng was born in 1981. He received the Ph.D. degree from the National Mobile Communications Research Laboratory, Southeast University in 2015. He is currently a lecturer at Jinling Institute of Technology. His research interests include cooperative communication, energy-efficient communication, physical layer security, and energy harvesting.

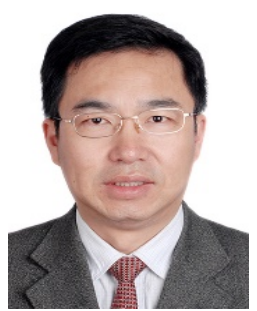

Wei Heng received the B.S. degree in information engineering from Huazhong University of Science and Technology in 1987. Then, he obtained the M.S. degree in signal and information processing and the Ph.D. degree in communications and information system from Southeast University in 1990 and 1997, respectively. From 1997 to 2002, he was a research scholar with the Catholic University of America. Since 2002, he has been a professor with the National Mobile Communications Research Laboratory, Southeast University. His research interests include mobile communications and signal processing. 